\title{
Dynamics of Micro-Air-Vehicle with Flapping Wings
}

K. Sibilski

Small (approximately 6 inch long, or hand-held) reconnaissance micro air vehicles (MAVs) will fly inside buildings, and require hover for observation, and agility at low speeds to move in confined spaces. For this flight envelope insect-like flapping wings seem to be an optimal mode of flying. Investigation of the aerodynamics of flapping wing MAVs is very challenging. The problem involves complex unsteady, viscous flow (mainly laminar), with the moving wing generating vortices and interacting with them. At this early stage of research only a preliminary insight into the nature of the little known aerodynamics of MAVs has been obtained. This paper describes computational models for simulation of the controlled motion of a microelectromechanical flying insect-entomopter. The design of software simulation for entomopter flight (SSEF) is presented. In particular, we will estimate the flight control algorithms and performance for a Micromechanical Flying Insect (MFI), a 80-100 mm (wingtip-to-wingtip) device capable of sustained autonomous flight. The SSEF is an end-to-end tool composed of several modular blocks which model the wing aerodynamics and dynamics, the body dynamics, and in the future, the environment perception, control algorithms, the actuators dynamics, and the visual and inertial sensors. We present the current state of the art of its implementation, and preliminary results.

Keywords: micromechanical flying insect, entomopter aerodynamics, entomopter dynamics, insect flight, software simulator, insect aerodynamics, insect dynamics.

\section{Introduction}

The development of small (approximately 6 inch long, or hand-held) autonomous flying vehicles is driven by the need for intelligent reconnaissance robots, capable of discreetly penetrating confined spaces, and manoeuvring in them without the assistance of a human telepilot [7]. This is particularly relevant to military operations in urban terrain. Flight inside buildings, stairwells, shafts and tunnels has significant military and civilian value, and requires agility at low speeds to avoid obstacles and move in confined spaces. The vehicles can be used in dull, dirty or dangerous (D3) environments, where direct or remote human assistance is not practical. Non-military uses will include law enforcement and rescue operations. The ability to explore D3 environments without human involvement will be of interest for many industries, for example, enabling air sampling in inaccessible areas, and examination of confined spaces in buildings, installations and large machines. The flight envelope of MAVs requires high agility (including hover) at low speeds (1-2 $\left.\mathrm{m} \mathrm{s}^{-1}\right)$ and silent flight, which is not easily met by scaled-down fixed or rotary wing aircraft. However, insect-like wing-flapping flight would appear to be very suitable for such applications requiring highly manoeuvrable flight through confined spaces.

Very recently, it has been recognized that flapping wing propulsion can be more efficient than conventional propellers if applied to MAVs, because of the very small Reynolds numbers encountered on such vehicles. Flapping flight is more complicated than flight with fixed or rotating wings. The key to understand the mechanisms of flapping flight is adequate physical and mathematical modeling. Animal propulsion by means of flapping wings was the focus of considerable interest in the late 1990s. This is due to the relatively high efficiency obtainable by such mode of flight. Flapping flight for micro-robots (known also as MAVs or micro-flyers) is not only an intriguing mode of locomotion but provides manoeuvrability not obtainable with fixed or even rotary wing aircraft. The MAV is comparable on size with small birds and large insects. In order to obtain satisfactory explanation of animal flight features, it is necessary to create adequate physical, mathematical and computational models. The key to this is to understand how the complex motions of animal wings generate aerodynamic forces. However, very little is still known about the flight dynamics and automatic control of flying micro-robots.

The unconventional aerodynamic concept associated with MAVs deserves a more detailed explanation. Insects fly by oscillating (frequency range: 5-200 Hz) and rotating their wings through large angles, which is possible because their wing articulation is not limited by an internal skeleton. The wing beat cycle can be divided into two distinct phases, the downstroke and the upstroke. At the beginning of downstroke the wing (as seen from the front of the insect) is in the uppermost position with the leading edge pointing forward. The wing is then pushed downwards and rotated continuously, resulting in large changes to the angle of attack. At the end of the downstroke the wing is twisted rapidly so that the leading edge points backwards, and the upstroke begins. During the upstroke the wing is pushed upwards and rotated again, changing the angle of attack throughout this phase. At the highest point the wing is twisted, so that the leading edge is pointing forwards again, and the next downstroke begins. Another important problem is the control of motion. Stabilising control is made difficult because the wings do not have typical control surfaces such as ailerons. Influence on the motion is possible only through changing the amplitudes and frequencies of flapping, lagging, and feathering of wings. The thrust of an entomopter depends on the local angles of attack, and these depend on the parameters of flapping and feathering.

In forward flight the downstroke lasts longer than the upstroke, because of the need to generate thrust in addition to lift. In the hover, where lift only is required, the two strokes are of equal duration. This mode of flying relies on unsteady aerodynamics [1], producing high lift coefficients (peak $C_{L}$ of the order of 3 is typical $[1,2,3]$ ), and excellent manoeuvrability. The unsteady mechanism varies with different insects, 
the most important being a bound leading edge vortex [4]. High lift is a major factor in high efficiency of the mechanism: a typical power requirement for insects is $30 \mathrm{~W} / \mathrm{kg}[2,11]$, whereas small, electrically-powered, propeller-driven, fixed wing aircraft require about $150 \mathrm{~W} / \mathrm{kg}$. Insect wing flapping occurs in a stroke plane that generally remains at the same orientation to the body, and may be horizontal or inclined. Rapid rotations occur at each end of the flapping half-stroke. To a first approximation, kinematic control of insect flight manoeuvres is provided by changes in the tilt of the stroke plane, which is analogous to helicopter control. Precise control is achieved by including inter-wing differences in the magnitude of the force produced, the timing of the downstroke-to-upstroke wing rotation, and the geometric position of the wings when the rotation occurs. The primarily goal of this work is to design the software simulation for a micromechanical flying insect (entomopter). The entomopter flight simulator should be an end-to-end tool composed of several modular blocks, which model: the wing aerodynamics, the body motion, and the control algorithms. We present the design of a software simulation for entomopter flight, the current state of the art of its implementation and preliminary results of calculations.

\section{The main issues}

\section{Features of airfoil for animals}

The typical airfoils for birds and bats are thin and cambered, which means that they generate very little leading-edge suction. Cruising birds and bats fly with their flapping axes aligned close to horizontal. This could produce an interesting dilemma for the upstroke. Insects have low-aspect-ratio wings, which are not suitable for cruising flight. During the downstroke, the insect generates mainly a vertical force. The acceleration of the insect's body during the first half of the downstroke is especially large, and this acceleration is mainly caused by a large unsteady pressure drag action on the wings. During the upstroke the insect generates mainly a horizontal force. The change of direction of the forces during the down-and-up-strokes is controlled by variations in the inclination of the stroke plane.

\section{Aerodynamic phenomena}

As the size of an aircraft is reduced, the need for efficiency in terms of lift and propulsion generation becomes more evident. Reducing the size of the lifting surfaces and keeping the flight speed around $15 \mathrm{~m} / \mathrm{s}$ makes the aerodynamic phenomena different from those found in normal size aircraft, mainly due to very low Reynolds number of the flow. Moreover, entomopter manoeuvring in this regime is subject to non-linear, unsteady aerodynamic loads [1, 2, 12]. The non-linearities and unsteadiness are due mainly to the large regions of 3-D separated flow and concentrated vortex flows that occur at large angles of attack. Accurate prediction of these non-linear, unsteady airloads is of great importance in the analysis of entomopter flight motion and in the design of its flight control system. Prediction of the unsteady airloads is complicated by the fact that the instantaneous flowfield surrounding a manoeuvring body, and thus the loading, is not determined solely by the instantaneous values of the motion variables, such as the angles of attack and sideslip, and, particularly in this study, by the deforming flexible wing parameters. In general, the instantaneous state of the flowfield depends on the time-history of the motion, that is, on all the states taken by the flowfield during the makeover prior to the instant in question.

\section{Degrees of freedom}

Not only at first sight, the study of entomopter flight dynamics and control may seem very complicated, since each wing possesses degrees of freedom in addition to those of the "fuselage". Detailed analyses of kinematics are central to an integrated understanding of animal flight. Four degrees of freedom in each wing are used to achieve flight in Nature: flapping, lagging, feathering, and spanning. Flapping is a rotation of the animal wing about the longitudinal axis of the animal body (this axis lies in the direction of the flight velocity), i.e. this is "up and down" motion. Lagging is a rotation about a "vertical" axis; this is the "forward and backward" wing motion backward parallel to the body. Feathering is an angular movement about the wing longitudinal axis (which may pass through the centre of gravity of the wing) which tilts the wing to change its angle of attack. Spanning is an expansion and contraction of the wingspan. Not all flying animals perform all of these motions. For instance insects with low wing flap frequencies about $20 \mathrm{~Hz}(17 \ldots 25 \mathrm{~Hz})$ generally have very restricted lagging capabilities. Unlike birds, most insects do not use the spanning technique. Insects such as alderfly (Apatele alni) and mayfly (Ephemera) have fixed stroke planes with respect to their bodies. Thus, flapping flight is possible with only two degrees of freedom: flapping and feathering. In the simplest physical models heaving and pitching represent these degrees of freedom. The motion of each bird wing may be decomposed into flapping, lagging, feathering (the rigid body motions) and also into more complex deflections of the surface from the base shape (vibration modes).

\section{Example of numerical calculations}

Sample results of calculations illustrating current capabilities of the method and providing a preliminary insight into the aerodynamic behaviour of flapping wings are shown in Fig. 1.

In order to evaluate the performance of flight control algorithms, a Software for Simulation of Entomopter Flight (SSEF) is being implemented to simulate the flight of an MFI inside a virtual environment. The SSEF is decomposed into several modular units, each of them responsible for an independent task. The Aerodynamic Module takes as its input the wing motion and the MFI body velocities, and gives as the output the corresponding aerodynamic forces and torques. This module corresponds to a mathematical model for the aerodynamics. The Body Dynamics Module takes the aerodynamic forces and torques generated by the wing kinematics and integrates them along with the dynamic model for the MFI body, thus computing the body's position and the attitude as a function of time.

The Control System Module takes as its input the MFI body state and eventually the perception of the external world. Its task is to decide a control strategy to achieve a desired mission and to generate the control signals to the electromechanical system. The electromechanical system 

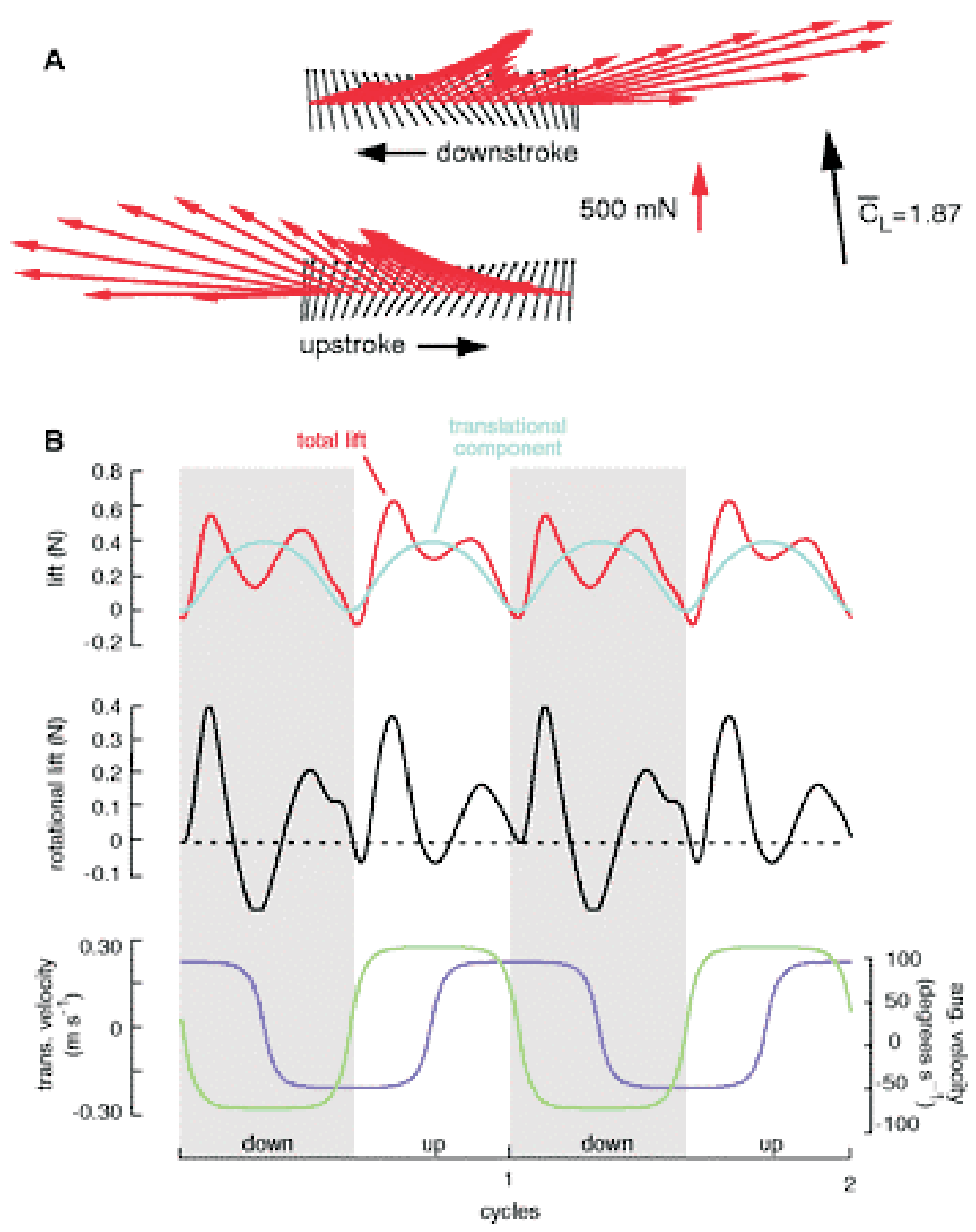

takes as its input the electrical control signals generated by the Control System Module and generates the corresponding wing kinematics. It consists of the model of the electromechanical wing-shorax architecture and the aerodynamic damping on the wings. The traces of the motion of the wings, the corresponding aerodynamic forces acting on them, and the trajectory motion and heading of the MFI produced by the SSEF, are combined together into a virtual environment simulation. The SSEF architecture is flexible, since it readily allows modifications or improvements of one single module without rewriting the whole simulator. For example, different combinations of control algorithms and electromechanical structure can be tested, giving rise to the more realistic setting of flight control with limited kinematics due to electromechanical constraints. Moreover, the dimensions and masses of the wings and body can be modified to analyze their effects on flight stability, power efficiency and maneuverability. Finally, as soon as better aerodynamic models are available, the aerodynamic module can be updated to improve accuracy. As present, the SSEF is not implemented: the Electromechanical System Modules, and the Sensory System Module. The following sections present the state of art for the SSEF.

\section{Aerodynamic modul}

Animal flapping flight represents an unusual aerodynamic problem because of the inherent "unsteadiness" and the low Reynolds number of the airflow. A large number of models for unsteady animal flight have been formulated, and these have been categorised and evaluated in a recent review by Smith et al [17], and Pietrucha et al [12].

Usually unsteady flow is defined as that in which the aerodynamic characteristics depend on time. Among various unsteady flows, linear, harmonic flows are of special importance. The linearity means that the amplitudes of the oscillations are small and that separation does not take place. For such flows it is sufficient that the aerodynamic characteristics are presented versus a frequency parameter. Time does appear explicit in the function describing these characteristics.

\section{Panel methods}

There are several methods for aerodynamic modelling by means of panel methods. In our opinion, the most valuable is the Unsteady Vortex Lattice Method. The Unsteady Vortex 
Lattice Method [6] employs an explicit routine for generating the unsteady wakes, instead of the implicit scheme requiring iteration. The wing may be viewed as moving through air either at rest or in motion. Thus, the effects of gusts on a manoeuvre can be modelled. The method is especially suitable for an impulsive start. However, giving the wing an impulsive start and then having it move at constant velocity until a steady state is developed can calculate all the steady flows. The method is based on the continuity equation with the few additional conditions. The method is especially valuable for analysing different manoeuvres, such as a steady turn, a fast roll about the wing longitudinal axis, response to aileron deflection, etc. To mark out the forces and aerodynamic moments effected on an entomopter's wing we have used the modified panel method [6, 17]. The choice of method was dictated by an easy application and low cost of calculations, which enables this problem to be realized on PC computers. Since the object has been found in unstationary motion, the solution has been found by the time-stepping method - which means that for every time step the wake vortex was suitably modified.

\section{Strip theory}

The classical strip theory approximation is based on the assumption that each element of a wing can be considered as an airfoil segment of a finite span wing. Lift and drag are then calculated from the resultant velocity acting on the airfoil, each element being considered independent of the adjoining elements. The aerodynamic characteristics of the wing are obtained by integrating the individual contribution of each element along the span. In order to obtain the resultant velocity at a wing element, the total flow over the wing must be known. It is composed of the resultant flight velocity, flapping velocity, and the induced velocity. A detailed explanation of the assumed formulas and algorithms can be found in [1], [2], and [9].

\section{Body dynamics module}

Given the aerodynamic forces generated by the wing kinematics, the Body Dynamics Module integrates the rigid body equations of motion, and gives the body position and attitude trajectories. The input to the body dynamics module is the stroke angle, lift and drag forces. It is a well-known fact that larger flying creatures fly principally by gliding or slow beating, where as smaller animals fly by strong beating at high frequency. Thus, the range of beating frequency and the Reynolds number varies greatly. The beating motion of the wings is exclusively used in the powered flight of birds and insects. In flying, this is the only way by means of the which these flying creatures can counter the gravity forces and propel themselves against aerodynamic drag. Therefore, detailed analyses of kinematics are central to an integrated understanding of animal flight $[1,2,4,8,9,11,12,13,14,15$, 16]. The motion of an animal wing may be decomposed into: flapping, lagging, feathering (the rigid body motions) and also into more complex deflections of the surface from the base shape (vibration modes). This requires a universal joint similar the shoulder in a human. A good model of such a joint is the articulated rotor hub. Flapping is a rotation of a wing about longitudinal axis of the body (this axis lies in the direction of flight velocity), i.e. "up and down" motion. Lagging is a rota- tion about a "vertical" axis, this is the "forward and backward" wing motion. Feathering is an angular movement about the wing longitudinal axis. During the feathering motion the wing changes its angle of attack. Spanning is an expanding and contracting of the wingspan. Not all flying animals implement all of these motions. Unlike birds, most insects do not use the spanning technique. Flapping flight is possible with only two degrees of freedom: flapping and feathering. In the simplest physical models heaving and pitching represent these degrees of freedom. This kind of motion can be generated principally by a flapping (up and down) motion of the wing, but not by a feathering (pitch-up and pitch-down) motion. The mode and frequency of the beating motion differ among different species and are strongly dependent on body size and shape. A typical difference in beating motion between birds and insects is observed in the way they use the aerodynamic forces, lift and drag. Birds rely entirely on lift because the Reynolds number of their wings is high enough. However, insects use drag as well as lift, thanks to the low Reynolds number and high frequency beating of low aspect ratio wings.

\section{Equations of entomopter motion}

\section{Gibbs-Appel equations}

The formalism of analytical mechanics allows us to present the dynamic equations of motion of an entomopter as mechanical systems in generalised co-ordinates. The method presented above provides a remarkably incredibly interesting and comfortable tool for constructing the equation of motion of an entomopter. Gibbs-Appel equations have the following form [16]:

$$
\frac{\mathrm{d}}{\mathrm{d} t}\left(\frac{\partial S}{\partial \ddot{\boldsymbol{q}}}\right)=\boldsymbol{Q}
$$

where: $\quad \boldsymbol{q}$-is the vector of generalised co-ordinates;

$S(\boldsymbol{q}, \dot{\boldsymbol{q}}, \ddot{\boldsymbol{q}}, t)-$ is the so called Appel function, or functional of accelerations.

Functional $S$ for the $i$-th element of the mechanical system is given by the equation [16]:

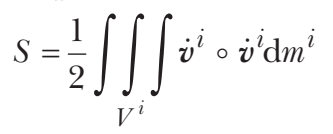

where $\dot{v}^{i}$ means the vector of absolute acceleration of elementary mass $\mathrm{d} m^{i}$ of the $i$-th body of the dynamical system considered (Fig. 2):

$$
\dot{\boldsymbol{v}}^{i}=\dot{\boldsymbol{v}}_{0}^{i}+\varepsilon_{0}^{i} \times \rho^{i}+\omega_{0}^{i} \times\left(\omega_{0}^{i} \times \rho^{i}\right) .
$$

Assuming that:

$$
\boldsymbol{r}^{i}=\boldsymbol{r}_{3}^{\prime i}+\boldsymbol{r}_{3}^{i}+\boldsymbol{r}_{3}^{i}
$$

and

$$
\begin{aligned}
& \boldsymbol{M}^{i}=\left[\begin{array}{cc}
m^{i} \boldsymbol{I} & m^{i} \widetilde{\boldsymbol{r}}^{i^{T}} \\
m \widetilde{\boldsymbol{r}}^{i} & \boldsymbol{J}_{0}^{i}
\end{array}\right] \\
& \boldsymbol{h}^{i}=\left[\begin{array}{c}
m^{i} \widetilde{\boldsymbol{\omega}}_{0}+m^{i} \widetilde{\boldsymbol{\omega}}_{0}^{2} \boldsymbol{r}^{i} \\
m^{i} \widetilde{\boldsymbol{r}}^{i} \widetilde{\boldsymbol{\omega}} \boldsymbol{v}_{0}^{i}+\widetilde{\boldsymbol{\omega}}_{0} \boldsymbol{J}_{0}^{i} \boldsymbol{\omega}_{0}
\end{array}\right]
\end{aligned}
$$


where $m^{i}$ is mass of the $\mathrm{i}$-th element, $\boldsymbol{J}_{0}^{i}$ tensor of inertia of the $i$-th element, $\omega_{0}$ vector of the angular velocity, $v_{0}^{i}$ vector of the velocity of the $i$-th element, and assuming that:

$$
\boldsymbol{a}=\left[a_{\xi}, a_{\eta}, a_{\zeta}\right]^{T}
$$

and

$$
\widetilde{\boldsymbol{a}}=\left[\begin{array}{ccc}
0 & -a_{\zeta} & a_{\eta} \\
a_{\eta} & 0 & -a_{\xi} \\
-a_{\eta} & a_{\xi} & 0
\end{array}\right] ;
$$

term (2) can be expressed in the following matrix form:

$$
S^{i}=\frac{1}{2}\left[\dot{\boldsymbol{v}}^{i}+\left(\boldsymbol{M}^{i}\right)^{-} \boldsymbol{h}^{i}\right]^{T} \boldsymbol{M}^{i}\left[\dot{\boldsymbol{v}}^{i}+\left(\boldsymbol{M}^{i}\right)^{-1} \boldsymbol{h}^{i}\right] .
$$

Calculating the matrixes $\boldsymbol{M}^{i}, \boldsymbol{h}^{i}$, the Appel function $S^{i}$ for all $k$ bodies of the system, and defining matrices:

$$
\begin{aligned}
& \boldsymbol{M}=\operatorname{diag}\left[\boldsymbol{M}^{1}, \boldsymbol{M}^{2}, \ldots, \boldsymbol{M}^{k}\right], \\
& \boldsymbol{v}=\left[\left(\boldsymbol{v}^{1}\right)^{T},\left(\boldsymbol{v}^{2}\right)^{T}, \ldots,\left(\boldsymbol{v}^{k}\right)^{T}\right]^{T}
\end{aligned}
$$

and

$$
\boldsymbol{h}=\left[\left(\boldsymbol{h}^{1}\right)^{T},\left(\boldsymbol{h}^{2}\right)^{T}, \ldots,\left(\boldsymbol{h}^{k}\right)^{T}\right]^{T},
$$

functional $S$ for the whole mechanical system is given by the equation:

$$
S=\frac{1}{2}\left(\dot{v}+M^{-1} h\right)^{T} \boldsymbol{M}\left(\dot{v}+M^{-1} \boldsymbol{h}\right)
$$

Assuming that $\boldsymbol{q}$ is the vector generalised coordinates of the mechanical system, the relations between $\boldsymbol{q}$ and $v$ are given by the equation:

$$
v=\boldsymbol{D}(\boldsymbol{q}, t) \dot{\boldsymbol{q}}+f(\boldsymbol{q}, t)
$$

hence:

$$
\dot{v}=\boldsymbol{D}(\boldsymbol{q}, t) \ddot{\boldsymbol{q}}+\varphi(\boldsymbol{q}, \dot{\boldsymbol{q}}, t)
$$

where:

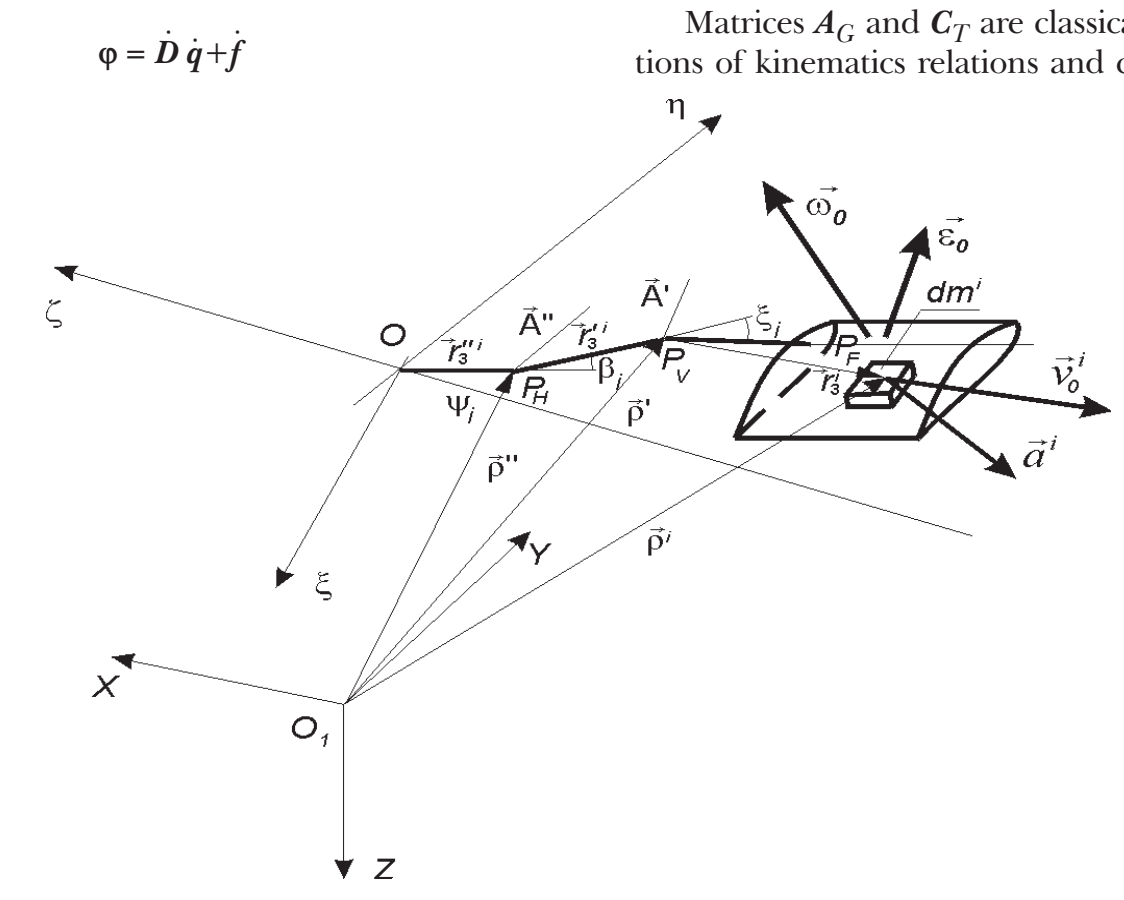

Fig. 2: Location of points, radius vectors and vectors of velocities and accelerations $\dot{w}=\left[\dot{w}_{1}, \dot{w}_{2}, \ldots, \dot{w}_{n}\right]$ is as follows:
Therefore the Appel function can be expressed by the following relation:

$S(\boldsymbol{q}, \dot{\boldsymbol{q}}, \ddot{\boldsymbol{q}}, t)=\frac{1}{2}\left(\boldsymbol{D} \ddot{\boldsymbol{q}}+\varphi+\boldsymbol{M}^{-1} \boldsymbol{h}\right)^{T} \boldsymbol{M}\left(\boldsymbol{D} \ddot{\boldsymbol{q}}+\varphi+\boldsymbol{M}^{-1} \boldsymbol{h}\right)$

Assuming that:

$\boldsymbol{M}_{g}=\boldsymbol{D}^{T} \boldsymbol{M D}$ and $\boldsymbol{h}_{g}=\boldsymbol{D}^{T}(\boldsymbol{M} \varphi+\boldsymbol{h})$

and remembering, that: $\boldsymbol{D}\left(\boldsymbol{D}^{T} \boldsymbol{M} \boldsymbol{D}\right)^{-1} \boldsymbol{D}^{T}=\boldsymbol{M}^{-1}$, equation (14) can be expressed in the form:

$$
S(\boldsymbol{q}, \dot{\boldsymbol{q}}, \ddot{\boldsymbol{q}}, t)=\frac{1}{2}\left(\ddot{\boldsymbol{q}}+\boldsymbol{M}_{g}^{-1} \boldsymbol{h}_{g}\right)^{T} \boldsymbol{M}\left(\ddot{\boldsymbol{q}}+\boldsymbol{M}_{g}^{-1} \boldsymbol{h}_{g}\right) .
$$

Non-linear equations of entomopter motion are expressed in a moving systems of co-ordinates [16]. In the case when we consider the model of a entomopter treated as a mechanical system containing a rigid fuselage and $n$ rigid wings fixed to the fuselage by means of three hinges, the vector generalised co-ordinates have the following form (Fig. 3):

$\boldsymbol{q}=\left[x_{s}, y_{s}, z_{s}, \Phi, \Theta, \Psi, \beta_{1}, \ldots, \beta_{n}, \zeta_{1}, \ldots, \zeta_{n}, \theta_{1}, \ldots, \theta_{n}\right]^{T}$.

The vector of quasi-velocities can be expressed by the following equation

$w=\left[u, v, w, p, q, r, \dot{\beta}_{1}, \ldots, \dot{\beta}_{n}, \dot{\zeta}_{1}, \ldots, \dot{\zeta}_{n}, \dot{\theta}_{1}, \ldots, \dot{\theta}_{n}\right]^{T}$.

For the holonomic dynamical system the relation between generalised velocities $\dot{\boldsymbol{q}}=\left[\dot{q}_{1}, \dot{q}_{2}, \ldots, \dot{q}_{n}\right]$ and quasi velocities

$$
\dot{q}=A_{T}(q) w
$$

Matrix $\boldsymbol{A}_{T}$ has a construction:

$$
\boldsymbol{A}_{T}=\left[\begin{array}{ccc}
\boldsymbol{A}_{G} & 0 & 0 \\
0 & \boldsymbol{C}_{T} & 0 \\
0 & 0 & \boldsymbol{I}
\end{array}\right]
$$

Matrices $\boldsymbol{A}_{G}$ and $\boldsymbol{C}_{T}$ are classical matrices of transformations of kinematics relations and can be found in [19], unit 


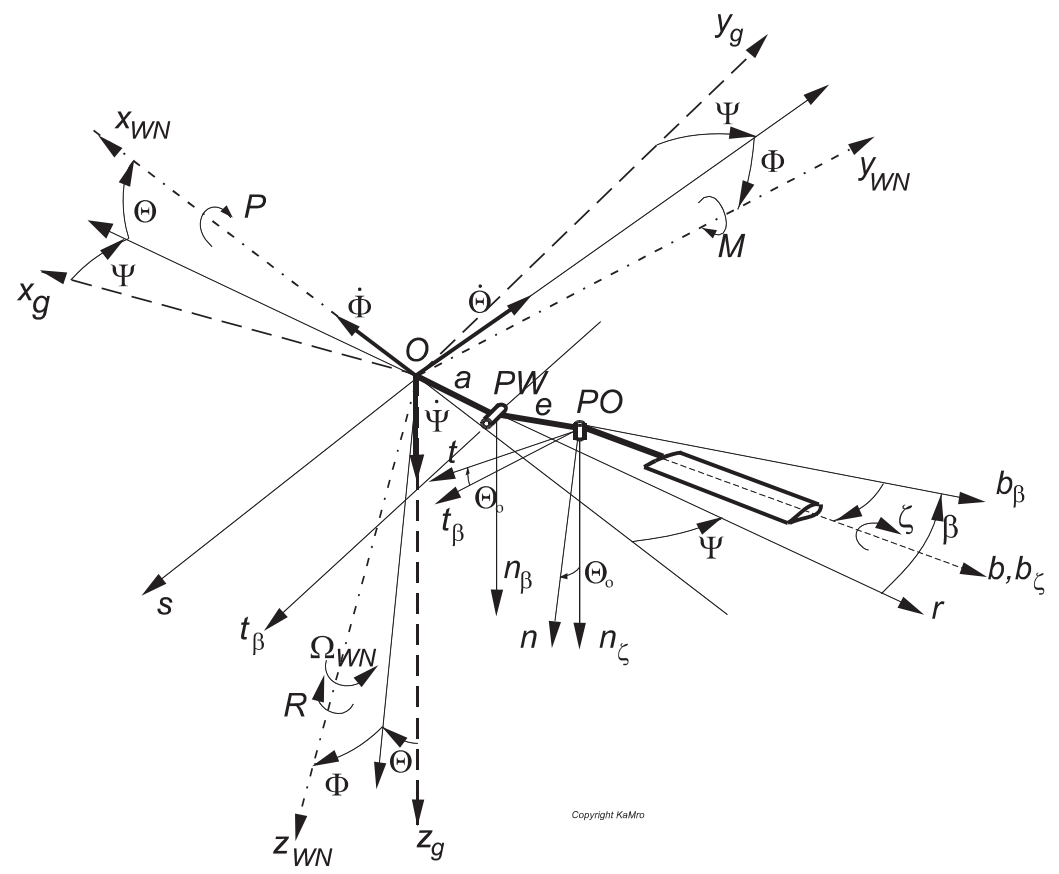

Fig. 3: Systems of coordinates

matrix $I$ has the dimension $(3 n+1) \times(3 n+1), n-$ number of main rotor blades.

From (18) we have the following relation:

$$
\ddot{\boldsymbol{q}}=A_{T}(\boldsymbol{q}) \dot{w}+\dot{A}_{T} w
$$

Finally, the Appel function has the following form:

$S^{*}(\boldsymbol{q}, \boldsymbol{w}, \dot{\boldsymbol{w}}, t)=\frac{1}{2}\left(\dot{\boldsymbol{w}}+\boldsymbol{M}_{w}^{-1} \boldsymbol{h}_{w}\right)^{T} \boldsymbol{M}_{w}\left(\dot{\boldsymbol{w}}+\boldsymbol{M}_{w}^{-1} \boldsymbol{h}_{w}\right)$

where $\boldsymbol{M}_{w}(\boldsymbol{q})=\boldsymbol{A}_{T}^{T} \boldsymbol{M}_{q} \boldsymbol{A}_{T}$,

and $\boldsymbol{h}_{w}(\boldsymbol{q}, \boldsymbol{w})=\boldsymbol{A}_{T}^{T}\left(\boldsymbol{M}_{q} \dot{\boldsymbol{A}}_{T}+\boldsymbol{h}_{q}\right)$.

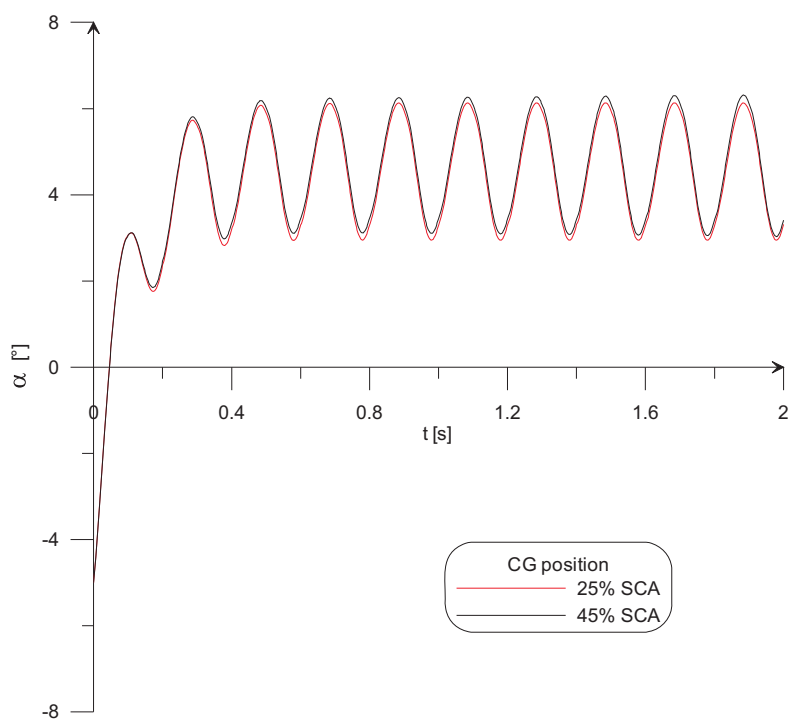

Gibbs-Appel equations of motion, written in quasi velocities, has the following form:

$$
\begin{aligned}
\left(\frac{\partial S^{*}}{\partial \dot{\boldsymbol{w}}}\right)^{T} & =\left[\frac{\partial S^{*}}{\partial \dot{w}_{1}}, \ldots, \frac{\partial S^{*}}{\partial \dot{w}_{k}}\right]^{T} \\
& =\boldsymbol{M}_{w}(\boldsymbol{q}, t) \dot{\boldsymbol{w}}+\boldsymbol{h}_{w}(\boldsymbol{q}, \boldsymbol{w}, t)=\boldsymbol{Q}^{*}(\boldsymbol{q}, \boldsymbol{w}, t)
\end{aligned}
$$

Vector $Q^{*}$ is the sum of aerodynamic loads, potential forces acting on the entomopter, and other non-potential forces acting on system Eq. (21).

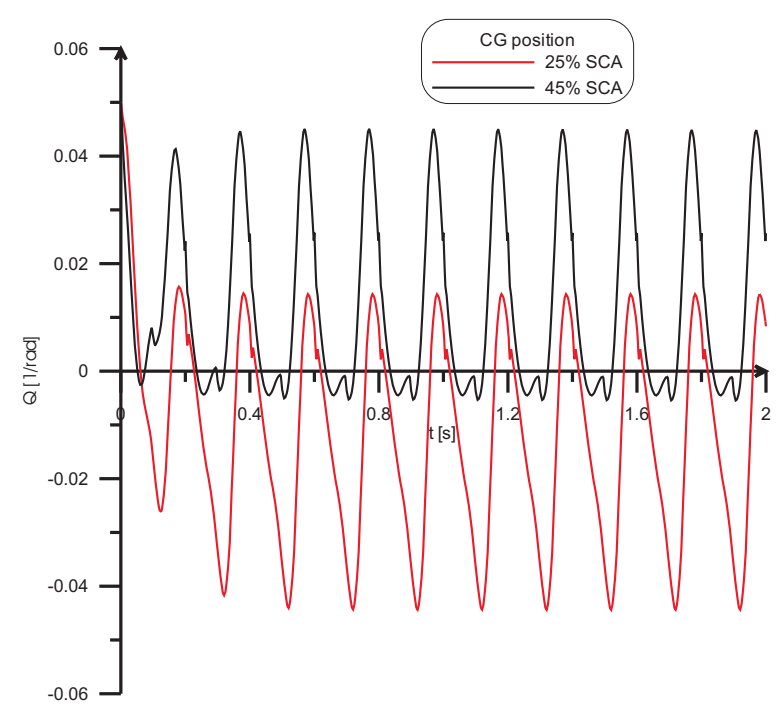

Fig. 4: Typical results of simulation 
Those equations are written in a form allowing the creation of procedures meant for their automatic formulation, (e.g., by means of such well known commercial software as Mathematica ${ }^{\circledR}$ or Mathcad $\left.{ }^{\circledR}\right)$.

\section{Results}

Sample calculations illustrating the current capabilities of the method and providing a preliminary insight into the behaviour of an entomopter are shown in Fig. 4. The dynamics of the entomopter shows an oscillatory motion superimposed on a vertical drifting term. The vertical drifting term is a result of a mean non-zero force along a wingbeat, while the oscillatory motion is the result of the time-varying nature of aerodynamic forces for insect flight.

\section{Conclusions}

This paper it is proposes the design of an accurate software simulation for entomopter flight that includes all major components involved: aerodynamics, body dynamics and control several of these components are modeled and implemented, and we have obtained simulation results that are consistent with observations of real flying animals (especially birds and bats). Finally, we plan to implement a 3D graphical visualization tool which can animate the motion of the simulated animalopter in a 3D environment. Current research is directed at improving some of the models considered, aerodynamic models and the control process, and to take advantage of this simulator to evaluate flight control schemes.

\section{References}

[1] Azuma A., Masato O., Kunio Y.: Aerodynamic characteristics of wings at low Reynolds Numbers. Fixed and flapping wings aerodynamics for micro air vehicle applications. Ed T. J. Mueller, Progress in Astronautics and Aeoronautics, AIAA, Reston, Va., 200, p. 341-398.

[2] Azuma A.: The biokinetics of flying and swimming. Springer Verlag, Tokyo, 1998.

[3] Ellington C. P.: "The aerodynamics of hovering insects flight. III Kinematics.” Philosophical Transactions of the Royal Society of London. Series B. Biological Sciences. Vol. 305, 1122, (1984), p. 41-78.

[4] Ellington C. P.: "The novel aerodynamics of insect flight: applications to micro-air-vehicles." The Journal of Experimental Biology. Vol. 202, (1999), p. 3439-3448.

[5] Gessow A., Myers G. C.: Aerodynamics of the helicopter. College Park Press, 1985.

[6] Goraj Z., Pietrucha J.: "Basic mathematical relations of fluid dynamics for modified panel methods." Journal of Theoretical and Applied Mechanics. Vol. 36, No. 1, (1998), p. 47-66.

[7] McMichael J. M., Francis M. S.: Micro Air Vehicles - Toward a New Generation of Flight:

http://www.darpa.mil/tto/mav/mav_auvsi.html
[8] Lasek M., Pietrucha J., Sibilski K., Złocka M.: Analogies between rotary and flapping wings from control theory point of view, AIAA Meeting papers on disc, Vol. 5, 3, AIAA 2001-4002 CP, 2001.

[9] Lasek M., Pietrucha J., Sibilski K., Złocka M.: A study of flight dynamics and automatic control of an animalopter, ICAS Paper no 339, Toronto, 2002.

[10] Lasek M., Pietrucha J., Sibilski K.: Micro Air Vehicle Manoeuvres as a Control Problem of Flexible Flapping Wings, AIAA Meeting Papers on Disc, Vol. 6, 1, (2002), AIAA 2002-0526 CP.

[11] Marusak A., Pietrucha J., Sibilski K., Złocka M.: Mathematical modelling of flying animals as aerial robot., $7^{\text {th }}$ IEEE Inter. Conf. on Methods and Models in Automation and Robotics (MMAR 2001), Międzyzdroje, Poland, Aug. 28-31, 2001.

[12] Pietrucha J., Sibilski K., Złocka M.: Modelling of aerodynamic forces on flapping wings - questions and results, Proc. of $4^{\text {th }}$ Inter. Seminary on RRDPAE-2000, Warsaw.

[13] Pornsin-Sisirak T., Lee S. W., Nassef H., Grasmeyer J., Tai Y. C., Ho C. M., Keenon M.: MEMS wing technology for a battery-powered ornithopter, $13^{\text {th }}$ IEEE Inter. Conf. On Micro Electro Mechanical Systems (MEMS '00), Miyazaki, Japan, Jan. 23-27 2000.

[14] Schenato L., Deng X., Wu W. C., Sastry S.: Virtual Insect Flight Simulator (VIFS): A Software Testbed of Insect Flight. IEEE International Conference on Robotics and Automation, 2001.

[15] Shyy W., Berg M., Ljungqvist D.: "Flapping and flexible wings for biological and micro air vehicles." Progress in Aerospace Sciences. Vol. 35, (1999), p. 455-505.

[16] Sibilski K. Modeling of an agile aircraft flight dynamics in limiting flight conditions. Military University of Technology, Warsaw, 1998.

[17] Smith M. J. C., Wilkin P. J., Williams M. H.: "The advantages of an unsteady panel method in modelling the aerodynamic forces on rigid flapping wings." Journal of Experimental Biology. Vol. 199 (1996), p. 1073-1083.

[18] Tobalski B. W., Dial K. P.: "Flight kinematics of black-billed magpies and pigeons over a wide range of speed." Journal of Experimental Biology. Vol. 199, (1996), p. 263-280.

[19] Willmott A. P., Ellington C. P.: "The Mechanics of Flight In The Hawkmoth Manduca Sexta. Part I. Kinematics of Hovering and Forward Flight." Journal of Experimental Biology. Vol. 200, (1997), p. 2705-2722.

\section{Krzysztof Sibilski}

Mechanical Engineering Department

Radom Technical University

Institute of Applied Mechanics

Malczewskiego 29

26-600 Radom, Poland 